\title{
Deviating arguments, impulsive effects, and positive solutions for second order singular $p$-Laplacian equations
}

Xuemei Zhang ${ }^{1 *}$ and Meiqiang Feng ${ }^{2}$

*Correspondence: zxm74@sina.com 1 Department of Mathematics and Physics, North China Electric Power University, Beijing, 102206, People's Republic of China

Full list of author information is available at the end of the article

\begin{abstract}
Using a new method for dealing with second order singular $p$-Laplacian equations with impulsive effects and deviating arguments, several new and more general results are obtained for the existence of at least single, twin or triple positive solutions by using Krasnosel'skii and Zabreiko's fixed point theorem, the fixed point theorem due to Avery and Henderson, and Leggett-Williams' fixed point theorem. We discuss our problems under two cases when the deviating arguments are delayed or advanced. Our results cover equations without deviating arguments and are compared with some recent results by Kajikiya, Lee, and Sim.
\end{abstract}

Keywords: deviating argument; impulse effect; second order singular $p$-Laplacian equation; cone and partial ordering

\section{Introduction}

Second order differential equations with $p$-Laplacian arise naturally in non-Newtonian mechanics, nonlinear elasticity, glaciology, population biology, combustion theory, and nonlinear flow laws; see $[1,2]$. In recent years many cases of the existence, multiplicity, and uniqueness of positive solution of differential equations with $p$-Laplacian have attracted considerable attention [3-20].

In [5], Kajikiya et al. investigated the following one-dimensional $p$-Laplacian problem:

$$
\left\{\begin{array}{l}
\left(\varphi_{p}\left(u^{\prime}\right)\right)^{\prime}+\lambda \omega(t) f(u)=0, \quad t \in(0,1) \\
u(0)=u(1)=0
\end{array}\right.
$$

and by virtue of the global bifurcation theory, they obtained the existence, nonexistence, uniqueness, and multiplicity of positive solutions as well as sign-changing solutions under suitable conditions imposed on the nonlinear $\operatorname{term} f$.

In [6], employing the shooting method, Iturriaga et al. obtained both existence and the exact number of positive solutions of the problem

$$
\left\{\begin{array}{l}
\lambda\left(\varphi_{p}\left(u^{\prime}\right)\right)^{\prime}+f(u)=0 \quad \text { in }(0,1), \\
u(0)=u(1)=0 .
\end{array}\right.
$$


Recently, using global bifurcation theory, Dai and Ma [8] showed the existence of nodal solutions for the following problem:

$$
\left\{\begin{array}{l}
\left(\varphi_{p}\left(u^{\prime}\right)\right)^{\prime}+f(t, u)=0, \quad t \in(0,1) \\
u(0)=u(1)=0
\end{array}\right.
$$

In [19], Ding and O'Regan studied a second order $p$-Laplacian differential equation involving the impulsive effect $\left.\Delta u\right|_{t=t_{k}}=I_{k}\left(u\left(t_{k}\right)\right), k=1,2, \ldots, m$. Using Jensen's inequality, the first eigenvalue of a relevant linear operator and the Krasnoselskii-Zabreiko fixed point theorem, the existence and multiplicity of positive solutions were established.

At the same time, a class of $p$-Laplacian differential equations with deviating arguments both of an advanced or delayed type have received much attention. For example, in [20], Jankowski considered the following third order $p$-Laplacian differential equation:

$$
\left\{\begin{array}{l}
\left(\varphi_{p}\left(u^{\prime \prime}(t)\right)\right)^{\prime}+h(t) f(t, u(t), u(\alpha(t)))=0, \quad t \in J^{\prime}, \\
u^{\prime}\left(t_{k}^{+}\right)=u^{\prime}\left(t_{k}^{-}\right)+Q_{k}\left(u\left(t_{k}\right)\right), \quad k=1,2, \ldots, m, \\
\beta u(0)-\gamma u^{\prime}(0)=0, \quad \delta u(1)+\eta u^{\prime}(1)=0, \quad u^{\prime \prime}(0)=0,
\end{array}\right.
$$

where $\alpha(t) \not \equiv t$ on $J$. The author obtained the existence of at least three positive solutions. The main tool is a fixed point theorem due to Avery [21], which is a generalization of the Leggett-Williams fixed point theorem.

Of course, a natural question is the following.

Q1. Can the existence of positive solutions for a second order $p$-Laplacian differential equation with deviating arguments both of an advanced or delayed type be proved?

Remark 1.1 In [20], by means of the properties of Green's function, Jankowski obtain the inequality

$$
\min _{t \in[\xi, 1]} u(t) \geq \rho\|u\|
$$

where $\xi \in(0,1)$ and $\rho \in(0,1)$.

In fact, the calculation of $\rho$ is very difficult when $t \in[\xi, 1]$. This is probably the main reason that there is almost no paper to study the existence of positive solutions for a class of second order $p$-Laplacian impulsive differential equations with two parameters and deviating arguments both of an advanced or delayed type. In [20], Jankowski obtained a constant number $\rho$ by means of the properties of Green's function. However, it is well known that there is not any Green's function whatsoever in one-dimensional $p$-Laplacian boundary value problems of second order differential equations. This implies the following question.

Q2. Can a similar inequality be obtained if there is no Green's function when $t \in[\xi, 1]$ ?

For this one needs to use a new technique to deal with second order $p$-Laplacian equations with deviating arguments, especially for second order $p$-Laplacian equations with impulsive effects.

Consider the one-dimensional $p$-Laplacian differential equation with deviating arguments

$$
-\left(\phi_{p}\left(u^{\prime}(t)\right)\right)^{\prime}=\omega(t) f(t, u(\alpha(t))), \quad t \neq t_{k}, t \in(0,1),
$$


subject to one of the following impulsive and boundary conditions:

$$
\left\{\begin{array}{l}
-\left.\Delta u\right|_{t=t_{k}}=I_{k}\left(u\left(t_{k}\right)\right), \quad k=1,2, \ldots, n, \\
u^{\prime}(0)=0, \quad u(1)=\int_{0}^{1} g(t) u(t) d t
\end{array}\right.
$$

or

$$
\left\{\begin{array}{l}
\left.\Delta u\right|_{t=t_{k}}=I_{k}\left(u\left(t_{k}\right)\right), \quad k=1,2, \ldots, n, \\
u(0)=\int_{0}^{1} h(t) u(t) d t, \quad u^{\prime}(1)=0,
\end{array}\right.
$$

where $\varphi_{p}(s)=|s|^{p-2} s, p>1,\left(\varphi_{p}\right)^{-1}=\varphi_{q}, \frac{1}{p}+\frac{1}{q}=1, \omega$ may be singular at $t=0$ and $/$ or $t=1, t_{k}$ $\left(k=1,2, \ldots, n\right.$, where $n$ is fixed positive integer) are fixed points with $0<t_{1}<t_{2}<\cdots<t_{k}<$ $\cdots<t_{n}<1,\left.\Delta u\right|_{t=t_{k}}$ denotes the jump of $u(t)$ at $t=t_{k}$, i.e.,

$$
\left.\Delta u\right|_{t=t_{k}}=u\left(t_{k}^{+}\right)-u\left(t_{k}^{-}\right)
$$

where $u\left(t_{k}^{+}\right)$and $u\left(t_{k}^{-}\right)$represent the right-hand limit and left-hand limit of $u(t)$ at $t=t_{k}$, respectively.

Throughout this paper we assume that $k=1,2, \ldots, n$ and $\alpha(t) \not \equiv t$ on $J=[0,1]$. In addition, $\omega, f, \alpha, I_{k}, g$, and $h$ satisfy

$\left(\mathrm{H}_{1}\right) \omega \in C((0,1),[0,+\infty))$ with $0<\int_{0}^{1} \omega(s) d s<\infty$ and $\omega$ does not vanish on any subinterval of $(0,1)$;

$\left(\mathrm{H}_{2}\right) f \in C([0,1] \times[0,+\infty),[0,+\infty)), \alpha \in C(J, J)$;

$\left(\mathrm{H}_{3}\right) I_{k} \in C([0,+\infty),[0,+\infty))$;

$\left(\mathrm{H}_{4}\right) g, h \in L^{1}[0,1]$ are nonnegative and $\sigma \in[0,1), \varsigma \in[0,1)$, where

$$
\sigma=\int_{0}^{1} g(s) d s, \quad \varsigma=\int_{0}^{1} h(s) d s .
$$

Some special cases of problem (1.1)-(1.2) or problem (1.1)-(1.3) have been investigated. For example, Kajikiya et al. [22] considered problem (1.1) in the case that $I_{k} \equiv 0, k=$ $1,2, \ldots, n$, and $\alpha(t) \equiv t$ on $J$. By using the properties of eigenfunctions and global bifurcation theory, the authors proved the existence, uniqueness, nonexistence, and multiplicity results of positive solutions as well as sign-changing solutions, specially when the nonlinear term is $p$-linear near 0 and $p$-sublinear at $\infty$.

Remark 1.2 There are almost no papers except [20, 23] studying one-dimensional $p$-Laplacian differential equations with deviating arguments both of an advanced or delayed type using fixed pointed theory. However, in [20,23], Jankowski only dealt with third order $p$-Laplacian equations and fourth order $p$-Laplacian equations. To our knowledge, it is the first paper where positive solutions have been investigated for the class of second order singular $p$-Laplacian equations with impulsive effects and deviating arguments both of an advanced or delayed type.

In this paper, we will give positive answers to Q1 and Q2. The organization of this paper is as follows. In Section 2, we first present some definitions and lemmas which are needed throughout this paper and then state several fixed point results: Krasnosel'skii and Zabreiko's fixed point theorem, a new fixed point theorem due to Avery, and Henderson 
and Leggett-Williams' fixed point theorem. In Section 3, we use a fixed point theorem of cone expansion and compression to obtain the existence of at least one or two positive solutions of problem (1.1)-(1.2) with advanced argument $\alpha$. Section 4 will further discuss the existence of twin positive solutions of problem (1.1)-(1.2) with advanced argument $\alpha$. Two new results will be presented by a new fixed point theorem due to Avery and Henderson. Section 5 is for developing existence criteria for (at least) three positive solutions of problem (1.1)-(1.2). Finally, in Section 6, we formulate sufficient conditions under which delayed problem (1.1)-(1.3) has at least one, two, and three positive solutions. In particular, our results in these sections are new when $\alpha(t) \equiv t$ on $t \in J$. To the best of the authors' knowledge, it is the first paper where the fixed point theories are applied to $p$-Laplacian boundary value problems of second order impulsive differential equations with deviating arguments.

\section{Preliminaries}

Let $J^{\prime}=J \backslash\left\{t_{1}, t_{2}, \ldots, t_{n}\right\}$ and $E$ be the Banach space

$$
E=\left\{u \mid u: J \rightarrow R \text { is continuous at } t \neq t_{k}, u\left(t_{k}^{-}\right)=u\left(t_{k}\right) \text { and } u\left(t_{k}^{+}\right) \text {exist, } k=1,2, \ldots, n\right\}
$$

with $\|u\|=\max _{0 \leq t \leq 1}|u(t)|$. We denote

$$
\Omega_{r}:=\{u \in E:\|u\|<r\}
$$

for all $r>0$ in the sequel.

In our main results, we will make use of the following definitions and lemmas.

Definition 2.1 (See [24]) Let $E$ be a real Banach space over $R$. A nonempty closed set $P \subset E$ is said to be a cone provided that

(i) $a u+b v \in P$ for all $u, v \in P$ and all $a \geq 0, b \geq 0$,

(ii) $u,-u \in P$ implies $u=0$.

Every cone $P \subset E$ induces an ordering in $E$ given by $x \leq y$ if and only if $y-x \in P$.

Definition 2.2 The map $\beta$ is said to be a nonnegative continuous concave functional on a cone $P$ of a real Banach space $E$ provided that $\beta: P \rightarrow[0, \infty)$ is continuous and

$$
\beta(t x+(1-t) y) \geq t \beta(x)+(1-t) \beta(y)
$$

for all $x, y \in P$ and $0 \leq t \leq 1$.

Definition 2.3 A function $u \in E \cap C^{1}(0,1)$ is called a solution of (1.1)-(1.2) if it satisfies (1.1)-(1.2). If $u(t) \geq 0$ and $u(t) \not \equiv 0$ on $J$, then $u$ is called a positive solution of (1.1)-(1.2).

Lemma 2.1 Assume that $\left(\mathrm{H}_{1}\right)-\left(\mathrm{H}_{4}\right)$ hold. Then $u \in E \cap C^{1}(0,1)$ is a solution of problem (1.1)-(1.2) if and only if $u \in E$ is a solution of the following equations:

$$
\begin{aligned}
u(t)= & \frac{1}{1-\sigma}\left\{\int_{0}^{1} g(t)\left[\int_{t}^{1} \phi_{q}\left(\int_{0}^{s} \omega(r) f(r, u(\alpha(r))) d r\right) d s\right] d t\right. \\
& \left.+\int_{0}^{1} g(t)\left(\sum_{t \leq t_{k}} I_{k}\left(u\left(t_{k}\right)\right)\right) d t\right\}
\end{aligned}
$$




$$
\begin{aligned}
& +\int_{t}^{1} \phi_{q}\left(\int_{0}^{s} \omega(r) f(r, u(\alpha(r))) d r\right) d s \\
& +\sum_{t \leq t_{k}} I_{k}\left(u\left(t_{k}\right)\right)
\end{aligned}
$$

and

$$
\min _{t \in[\xi, 1]} u(t) \geq \delta\|u\|
$$

where $0<\xi<t_{1}$ and

$$
\delta=\frac{(1-\xi) \int_{\xi}^{1}(1-t) g(t) d t}{1-\xi-(1-\xi) \int_{\xi}^{1} g(t) d t+\int_{\xi}^{1}(1-t) g(t) d t} .
$$

Proof First suppose that $u \in E \cap C^{1}(0,1)$ is a solution of problem (1.1)-(1.2). It is easy to see by integration of (1.1) that

$$
-\phi_{p}\left(u^{\prime}(t)\right)+\phi_{p}\left(u^{\prime}(0)\right)=\int_{0}^{t} \omega(s) f(s, u(\alpha(s))) d s .
$$

By the boundary condition, we have

$$
u^{\prime}(t)=-\phi_{q}\left(\int_{0}^{t} \omega(s) f(s, u(\alpha(s))) d s\right)
$$

If $t_{n-1}<t<t_{n}$, then integrating (2.4) from $t_{n}$ to 1 , we have

$$
u(1)-u\left(t_{n}^{+}\right)=-\int_{t_{n}}^{1} \phi_{q}\left(\int_{0}^{s} \omega(r) f(r, u(\alpha(r))) d r\right) d s
$$

Integrating (2.4) from $t$ to $t_{n}$, we have

$$
u\left(t_{n}^{-}\right)-u(t)=-\int_{t}^{t_{n}} \phi_{q}\left(\int_{0}^{s} \omega(r) f(r, u(\alpha(r))) d r\right) d s .
$$

So, (2.5) and (2.6) imply

$$
u(1)-u(t)=-\int_{t}^{1} \phi_{q}\left(\int_{0}^{s} \omega(r) f(r, u(\alpha(r))) d r\right) d s-I_{n}\left(u\left(t_{n}\right)\right), \quad t_{n-1}<t<t_{n} .
$$

Repeating the above procession, for $t \in J$, we have

$$
u(t)=u(1)+\int_{t}^{1} \phi_{q}\left(\int_{0}^{s} \omega(r) f(r, u(\alpha(r))) d r\right) d s+\sum_{t \leq t_{k}} I_{k}\left(u\left(t_{k}\right)\right) .
$$

Substituting $u(1)=\int_{0}^{1} g(t) u(t) d t$ into (2.7), we obtain

$$
u(t)=\int_{0}^{1} g(t) u(t) d t+\int_{t}^{1} \phi_{q}\left(\int_{0}^{s} \omega(r) f(r, u(\alpha(r))) d r\right) d s+\sum_{t \leq t_{k}} I_{k}\left(u\left(t_{k}\right)\right) .
$$


Then we have

$$
\begin{aligned}
\int_{0}^{1} g(t) u(t) d t= & \int_{0}^{1} g(t) u(t) d t \times \sigma+\int_{0}^{1} g(t)\left(\sum_{t \leq t_{k}} I_{k}\left(u\left(t_{k}\right)\right)\right) d t \\
& +\int_{0}^{1} g(t)\left[\int_{t}^{1} \phi_{q}\left(\int_{0}^{s} \omega(r) f(r, u(\alpha(r))) d r\right) d s\right] d t
\end{aligned}
$$

and

$$
\begin{aligned}
u(t)= & \frac{1}{1-\sigma}\left\{\int_{0}^{1} g(t)\left[\int_{t}^{1} \phi_{q}\left(\int_{0}^{s} \omega(r) f(r, u(\alpha(r))) d r\right) d s\right] d t\right. \\
& \left.+\int_{0}^{1} g(t)\left(\sum_{t \leq t_{k}} I_{k}\left(u\left(t_{k}\right)\right)\right) d t\right\} \\
& +\int_{t}^{1} \phi_{q}\left(\int_{0}^{s} \omega(r) f(r, u(\alpha(r))) d r\right) d s+\sum_{t \leq t_{k}} I_{k}\left(u\left(t_{k}\right)\right) .
\end{aligned}
$$

Then the proof of sufficiency is complete.

Conversely, we see if $u \in E$ is a solution of (2.1).

Direct differentiation of (2.1) implies, for $t \neq t_{k}$,

$$
u^{\prime}(t)=-\phi_{q}\left(\int_{0}^{t} \omega(s) f(s, u(\alpha(s))) d s\right) .
$$

Evidently,

$$
\begin{aligned}
& \left(\phi_{p}\left(u^{\prime}(t)\right)\right)^{\prime}=-f(t, u(\alpha(t))), \\
& \left.\Delta u\right|_{t=t_{k}}=-I_{k}\left(u\left(t_{k}\right)\right) \quad(k=1,2, \ldots, n), \quad u^{\prime}(0)=0, \quad u(1)=\int_{0}^{1} g(t) u(t) d t .
\end{aligned}
$$

Finally, we show that (2.2) and (2.3) hold. It is clear that $u^{\prime}(t)=-\phi_{q}\left(\int_{0}^{t} f(s, u(\alpha(s))) d s\right)<0$, which implies that

$$
\|u\|=u(0), \quad \min _{t \in[0,1]} u(t)=u(1) .
$$

On the other hand, for given $s_{1}, s_{2} \in J^{\prime}$ with $s_{1} \leq s_{2}$, one can prove that $u^{\prime}\left(s_{2}\right) \leq u^{\prime}\left(s_{1}\right)$. Hence, $u^{\prime}(t)$ is nonincreasing on $J^{\prime}$.

So, for every $t \in[\xi, 1)$, we have

$$
\frac{u(\xi)-u(1)}{1-\xi} \leq \frac{u(t)-u(1)}{1-t}
$$

i.e., $(1-\xi)(u(t)-u(1)) \geq(1-t)[u(\xi)-u(1)]$.

Therefore,

$$
\begin{aligned}
& (1-\xi) \int_{\xi}^{1} g(t) u(t) d t-\int_{\xi}^{1}(1-\xi) g(t) u(1) d t \\
& \quad \geq u(\xi) \int_{\xi}^{1}(1-t) g(t) d t-\int_{\xi}^{1}(1-t) g(t) u(1) d t .
\end{aligned}
$$


This together with the boundary condition $u(1)=\int_{0}^{1} g(t) u(t) d t$ implies that

$$
u(1) \geq \frac{\int_{\xi}^{1}(1-t) g(t) d t}{1-\xi-(1-\xi) \int_{\xi}^{1} g(t) d t+\int_{\xi}^{1}(1-t) g(t) d t} u(\xi) .
$$

It is clear that (2.8) holds when $t=1$.

Noticing that

$$
\frac{u(0)-u(1)}{1} \leq \frac{u(\xi)-u(1)}{1-\xi}
$$

we have

$$
u(\xi) \geq \xi u(1)+(1-\xi) u(0) \geq(1-\xi) u(0) .
$$

It follows from (2.8) and (2.9) that (2.2) and (2.3) hold.

Define a cone $K$ in $E$ by

$$
K=\left\{u \in E: u \geq 0, \min _{t \in[\xi, 1]} u(t) \geq \delta\|u\|\right\}
$$

where $\delta$ is defined in (2.3). It is easy to see that $K$ is a closed convex cone of $E$.

Define $T: K \rightarrow E$ by

$$
\begin{aligned}
(T u)(t)= & \frac{1}{1-\sigma}\left\{\int_{0}^{1} g(t)\left[\int_{t}^{1} \phi_{q}\left(\int_{0}^{s} \omega(r) f(r, u(\alpha(r))) d r\right) d s\right] d t\right. \\
& \left.+\int_{0}^{1} g(t)\left(\sum_{t \leq t_{k}} I_{k}\left(u\left(t_{k}\right)\right)\right) d t\right\} \\
& +\int_{t}^{1} \phi_{q}\left(\int_{0}^{s} \omega(r) f(r, u(\alpha(r))) d r\right) d s+\sum_{t \leq t_{k}} I_{k}\left(u\left(t_{k}\right)\right) .
\end{aligned}
$$

Obviously, $(T u)(t) \geq 0$ for $t \in J$.

From the definition of $T$ and the proof of Lemma 2.1, we claim that for each $u \in K$, $T u \in K$ and satisfies (1.1), and $(T u)(0)$ is the maximum value of $(T u)(t)$ on $J$.

From (2.10) and Lemma 2.1, it is also easy to obtain the following results.

Lemma 2.2 Assume that $\left(\mathrm{H}_{1}\right)-\left(\mathrm{H}_{4}\right)$ hold. Then problem (1.1)-(1.2) is equivalent to the fixed point problem of $T$ in $K$.

Lemma 2.3 Assume that $\left(\mathrm{H}_{1}\right)-\left(\mathrm{H}_{4}\right)$ hold. Then $T: K \rightarrow K$ is completely continuous.

Lemma 2.4 (Fixed point theorem of cone expansion and compression; see [24]) Let $\Omega_{1}$ and $\Omega_{2}$ be two bounded open sets in Banach space E, such that $0 \in \Omega_{1}$ and $\bar{\Omega}_{1} \subset \Omega_{2}$. Let $P$ be a cone in $E$ and let operator $A: P \cap\left(\bar{\Omega}_{2} \backslash \Omega_{1}\right) \rightarrow P$ be completely continuous. Suppose that one of the following two conditions is satisfied:

(i) $A x \nsupseteq x, \forall x \in P \cap \partial \Omega_{1} ; A x \not \leq x, \forall x \in P \cap \partial \Omega_{2}$

and 
(ii) $A x \not \leq x, \forall x \in P \cap \partial \Omega_{1} ; A x \nsupseteq x, \forall x \in P \cap \partial \Omega_{2}$.

Then $A$ has at least one fixed point in $P \cap\left(\Omega_{2} \backslash \bar{\Omega}_{1}\right)$.

Remark 2.1 To the best of our knowledge, it is the first paper where the fixed point theorem of cone expansion and compression is applied to $p$-Laplacian differential equations.

Given a nonnegative continuous functional $\psi$ on a cone $P$ of a real Banach space, we define, for each $r>0$, the set

$$
P(\psi, r)=\{u \in P: \psi(u)<r\} .
$$

Lemma 2.5 (See [25]) Let $P$ be a cone in a real Banach space. Let $\alpha$ and $\gamma$ be increasing nonnegative continuous functional on $P$, and let $\theta$ be a nonnegative continuous functional on $P$ with $\theta(0)=0$ such that, for some $c>0$ and $H>0$,

$$
\gamma(x) \leq \theta(x) \leq \alpha(x) \quad \text { and } \quad\|x\| \leq H \gamma(x)
$$

for all $x \in \overline{P(\gamma, c)}$. Suppose there exist a completely continuous operator $A: \overline{P(\gamma, c)} \rightarrow P$ and $0<a<b<c$ such that

$$
\theta(\lambda x) \leq \lambda \theta(x) \quad \text { for } 0 \leq \lambda \leq 1 \text { and } x \in \partial P(\theta, b)
$$

and

(i) $\gamma(A x)>$ for all $x \in \partial P(\gamma, c)$;

(ii) $\theta(A x)<b$ for all $x \in \partial P(\theta, b)$;

(iii) $P(\alpha, a) \neq \emptyset$ and $\alpha(A x)>a$ for $x \in \partial P(\alpha, a)$.

Then $A$ has at least two positive solutions $x_{1}$ and $x_{2}$ belonging to $\overline{P(\gamma, c)}$ satisfying

$$
a<\alpha\left(x_{1}\right) \text { with } \theta\left(x_{1}\right)<b \text { and } b<\theta\left(x_{2}\right) \text { with } \gamma\left(x_{2}\right)<c \text {. }
$$

Let $0<a<b$ be given and let $\beta$ be a nonnegative continuous concave functional on the cone $K$. Define the convex sets $K_{a}, K(\beta, a, b)$ by

$$
\begin{aligned}
& K_{a}=\{x \in K:\|x\|<a\}, \\
& K(\beta, a, b)=\{x \in K: a \leq \beta(x),\|x\| \leq b\} .
\end{aligned}
$$

Finally we state the Leggett-Williams fixed point theorem [26].

Lemma 2.6 (See [26]) Let $K$ be a cone in a real Banach space $E, A: \bar{K}_{a} \rightarrow \bar{K}_{a}$ be completely continuous and $\beta$ be a nonnegative continuous concave functional on $K$ with $\beta(x) \leq\|x\|$ for all $x \in K_{a}$. Suppose there exist $0<d<a<b \leq c$ such that

(i) $\{x \in K(\beta, a, b): \beta(x)>a\} \neq \emptyset$ and $\beta(A x)>a$ for $x \in K(\beta, a, b)$;

(ii) $\|A x\|<d$ for $\|x\| \leq d$;

(iii) $\beta(A x)>a$ for $x \in K(\beta, a, c)$ with $\|A x\|>b$.

Then $A$ has at least three positive solutions, $x_{1}, x_{2}, x_{3}$, satisfying

$$
\left\|x_{1}\right\|<d, \quad a<\beta\left(x_{2}\right), \quad\left\|x_{3}\right\|>d \quad \text { and } \quad \beta\left(x_{3}\right)<a .
$$




\section{Single or twin solutions of problem (1.1)-(1.2) for the case $\alpha(t) \geq t$ on $J$}

For convenience we introduce the following notations:

$$
\begin{aligned}
& f_{\delta \rho}^{\rho}=\min \left\{\min _{t \in[\xi, 1]} \frac{f(t, u)}{\phi_{p}(\rho)}: u \in[\delta \rho, \rho]\right\}, \quad I_{\delta \rho}^{\rho}(k)=\min \left\{\frac{I_{k}(u)}{\rho}: u \in[\delta \rho, \rho]\right\}, \\
& f_{0}^{\rho}=\max \left\{\max _{t \in J} \frac{f(t, u)}{\phi_{p}(\rho)}: u \in[0, \rho]\right\}, \quad I_{0}^{\rho}(k)=\max \left\{\frac{I_{k}(u)}{\rho}: u \in[0, \rho]\right\}, \\
& f^{\varrho}=\limsup _{u \rightarrow \varrho} \max _{t \in[0,1]} \frac{f(t, u)}{\phi_{p}(u)}, \quad I^{\varrho}(k)=\limsup _{u \rightarrow \varrho} \frac{I_{k}(u)}{u}, \\
& f_{\varrho}=\liminf _{u \rightarrow \varrho} \min _{t \in[\xi, 1]} \frac{f(t, u)}{\phi_{p}(u)}, \quad I_{\varrho}(k)=\liminf _{u \rightarrow \varrho} \frac{I_{k}(u)}{u} \quad\left(\varrho:=\infty \text { or } 0^{+}, k=1,2, \ldots, n\right), \\
& \gamma=\varphi_{q}\left(\int_{0}^{1} \omega(s) d s\right), \quad \sigma_{1}=\int_{\xi}^{t_{n}} g(s) d s, \\
& \frac{1}{l}=\frac{\gamma+n}{1-\sigma}, \quad \frac{1}{L}=\frac{\sigma_{1}}{1-\sigma} .
\end{aligned}
$$

Remark 3.1 It follows from the definitions of $l$ and $L$ that $L>l$.

Theorem 3.1 Suppose $\left(\mathrm{H}_{1}\right)-\left(\mathrm{H}_{4}\right)$ hold, $\alpha(t) \geq t$ on $J$ and there exist $\rho_{1}, \rho_{2} \in(0, \infty)$ with $\rho_{1}<\delta \rho_{2}$ such that the following conditions hold:

$\left(\mathrm{H}_{5}\right) f_{0}^{\rho_{1}}<\phi_{p}(l), I_{0}^{\rho_{1}}(k)<l$.

$\left(\mathrm{H}_{6}\right) I_{\delta \rho_{2}}^{\rho_{2}}(k) \geq L$.

Then problem (1.1)-(1.2) has at least one positive solution $u$ with $\rho_{1}<\|u\| \leq \rho_{2}$.

Proof Considering $\left(\mathrm{H}_{5}\right)$, we have $f(t, x) \leq \phi_{p}\left(l \rho_{1}\right), I_{k}(x) \leq l \rho_{1}$ for $0 \leq x \leq \rho_{1}, t \in J$.

Since $0 \leq t \leq \alpha(t) \leq 1$ on $J$, it follows from $0 \leq x(t) \leq \rho_{1}$ on $J$ that

$$
0 \leq x(\alpha(t)) \leq \rho_{1}
$$

Next, we prove that

$$
T u \nsupseteq u, \quad u \in K, \quad\|u\|=\rho_{1} .
$$

In fact, if there exists $u_{1} \in K,\left\|u_{1}\right\|=\rho_{1}$ such that $T u_{1} \geq u_{1}$, then we have

$$
\begin{aligned}
\max _{t \in J} u_{1}(t) \leq & \max _{t \in J}\left\{\frac { 1 } { 1 - \sigma } \left\{\int_{0}^{1} g(t)\left[\int_{t}^{1} \phi_{q}\left(\int_{0}^{s} \omega(r) f\left(r, u_{1}(\alpha(r))\right) d r\right) d s\right] d t\right.\right. \\
& \left.+\int_{0}^{1} g(t)\left(\sum_{t \leq t_{k}} I_{k}\left(u_{1}\left(t_{k}\right)\right)\right) d t\right\} \\
& \left.+\int_{t}^{1} \phi_{q}\left(\int_{0}^{s} \omega(r) f\left(r, u_{1}(\alpha(r))\right) d r\right) d s+\sum_{t \leq t_{k}} I_{k}\left(u_{1}\left(t_{k}\right)\right)\right\} \\
\leq & \frac{1}{1-\sigma}\left\{\int_{0}^{1} g(t)\left[\int_{0}^{1} \phi_{q}\left(\int_{0}^{s} \omega(r) f\left(r, u_{1}(\alpha(r))\right) d r\right) d s\right] d t\right.
\end{aligned}
$$




$$
\begin{aligned}
& \left.+\int_{0}^{1} g(t)\left(\sum_{k=1}^{n} I_{k}\left(u_{1}\left(t_{k}\right)\right)\right) d t\right\} \\
& +\int_{0}^{1} \phi_{q}\left(\int_{0}^{s} \omega(r) f\left(r, u_{1}(\alpha(r))\right) d r\right) d s+\sum_{k=1}^{n} I_{k}\left(u_{1}\left(t_{k}\right)\right) \\
= & \frac{1}{1-\sigma} \int_{0}^{1} \phi_{q}\left(\int_{0}^{s} \omega(r) f\left(r, u_{1}(\alpha(r))\right) d r\right) d s+\frac{1}{1-\sigma} \sum_{k=1}^{n} I_{k}\left(u_{1}\left(t_{k}\right)\right) \\
\leq & \frac{1}{1-\sigma} \int_{0}^{1} \phi_{q}\left(\int_{0}^{1} \omega(r) f\left(r, u_{1}(\alpha(r))\right) d r\right) d s+\frac{1}{1-\sigma} \sum_{k=1}^{n} I_{k}\left(u_{1}\left(t_{k}\right)\right) \\
< & \frac{1}{1-\sigma} \phi_{q}\left(\int_{0}^{1} \omega(r) \phi_{p}\left(l \rho_{1}\right) d r\right)+\frac{1}{1-\sigma} \sum_{k=1}^{n} l \rho_{1} \\
= & \frac{l \rho_{1}}{1-\sigma} \phi_{q}\left(\int_{0}^{1} \omega(r) d r\right)+\frac{1}{1-\sigma} \sum_{k=1}^{n} l \rho_{1} \\
= & \frac{l \rho_{1}}{1-\sigma} \gamma+\frac{n}{1-\sigma} l \rho_{1} \\
= & \rho_{1}=\left\|u_{1}\right\| .
\end{aligned}
$$

This implies $\left\|u_{1}\right\|<\left\|u_{1}\right\|$, which is a contraction. Hence (3.1) holds.

Considering $\left(\mathrm{H}_{6}\right)$, we have $I_{k}(x) \geq L \rho_{2}$ for $\delta \rho_{2} \leq x \leq \rho_{2}$.

Next, we prove that

$$
T u \not \leq u, \quad u \in K, \quad\|u\|=\rho_{2} .
$$

In fact, if there exists $u_{0} \in K,\left\|u_{0}\right\|=\rho_{2}$ such that $T u_{0} \leq u_{0}$, then we have

$$
\delta \rho_{2} \leq u_{0} \leq \rho_{2}
$$

for $t \in[\xi, 1]$, and then

$$
\begin{aligned}
0 \leq t \in J \Rightarrow u_{0}(t) \geq & \left(T u_{0}\right)(t) \\
= & \frac{1}{1-\sigma}\left\{\int_{0}^{1} g(t)\left[\int_{t}^{1} \phi_{q}\left(\int_{0}^{s} \omega(r) f\left(r, u_{0}(\alpha(r))\right) d r\right) d s\right] d t\right. \\
& \left.+\int_{0}^{1} g(t)\left(\sum_{t \leq t_{k}} I_{k}\left(u_{0}\left(t_{k}\right)\right)\right) d t\right\} \\
& +\int_{t}^{1} \phi_{q}\left(\int_{0}^{s} \omega(r) f\left(r, u_{0}(\alpha(r))\right) d r\right) d s+\sum_{t \leq t_{k}} I_{k}\left(u_{0}\left(t_{k}\right)\right) \\
\geq & \frac{1}{1-\sigma} \int_{0}^{1} g(t)\left(\sum_{t \leq t_{k}} I_{k}\left(u_{0}\left(t_{k}\right)\right)\right) d t \\
\geq & \frac{1}{1-\sigma}\left[\int_{t_{n-1}}^{t_{n}} g(t) I_{n}\left(u_{0}\left(t_{n}\right)\right) d t\right. \\
& +\int_{t_{n-2}}^{t_{n-1}} g(t)\left(I_{n-1}\left(u_{0}\left(t_{n-1}\right)\right)+I_{n}\left(u_{0}\left(t_{n}\right)\right)\right) d t+\cdots
\end{aligned}
$$




$$
\begin{aligned}
& \left.+\int_{0}^{t_{1}} g(t)\left(I_{1}\left(u_{0}\left(t_{1}\right)\right)+I_{2}\left(u_{0}\left(t_{2}\right)\right)+\cdots+I_{n}\left(u_{0}\left(t_{n}\right)\right)\right) d t\right] \\
= & \frac{1}{1-\sigma}\left[\int_{0}^{t_{n}} g(t) I_{n}\left(u_{0}\left(t_{n}\right)\right) d t\right. \\
& \left.+\int_{0}^{t_{n-1}} g(t) I_{n-1}\left(u_{0}\left(t_{n-1}\right)\right) d t+\cdots+\int_{0}^{t_{1}} g(t) I_{1}\left(u_{0}\left(t_{1}\right)\right) d t\right] \\
\geq & \frac{1}{1-\sigma} \int_{0}^{t_{n}} g(t) I_{n}\left(u_{0}\left(t_{n}\right)\right) d t \\
\geq & \frac{1}{1-\sigma} \int_{\xi}^{t_{n}} g(t) I_{n}\left(u_{0}\left(t_{n}\right)\right) d t \\
= & \frac{1}{1-\sigma} L \rho_{2} \sigma_{1} \\
= & \rho_{2}=\left\|u_{0}\right\| .
\end{aligned}
$$

This is also a contraction. Hence (3.2) holds.

Applying (i) of Lemma 2.4 to (3.1) and (3.2) shows that $T$ has a fixed point $u \in K \cap$ $\left(\Omega_{\rho_{2}} \backslash \bar{\Omega}_{\rho_{1}}\right)$. Thus, it follows that problem (1.1)-(1.2) has one positive solution $u$ satisfying $\rho_{1}<\|u\| \leq \rho_{2}$. This gives the proof of the theorem.

Noticing that $L>l$, it follows from the proof of Theorem 3.1 that:

Corollary 3.1 Suppose $\left(\mathrm{H}_{1}\right)-\left(\mathrm{H}_{4}\right)$ hold, $\alpha(t) \geq t$ on $J$ and there exist $\rho_{1}, \rho_{2} \in(0, \infty)$ with $L \rho_{2}<l \rho_{1}$ such that $\left(\mathrm{H}_{5}\right)$ and $\left(\mathrm{H}_{6}\right)$ hold. Then problem (1.1)-(1.2) has at least one positive solution $u$ with $\rho_{2}<\|u\| \leq \rho_{1}$.

Corollary 3.2 Suppose $\left(\mathrm{H}_{1}\right)-\left(\mathrm{H}_{4}\right)$ hold, $\alpha(t) \geq t$ on $J$ and there exist $\rho_{1}, \rho_{2} \in(0, \infty)$ with $\rho_{1}<\rho_{2}$ such that the following conditions hold:

$\left(\mathrm{H}_{5}^{*}\right) f_{0}^{\rho_{2}}<\phi_{p}(l), I_{0}^{\rho_{2}}(k)<l$.

$\left(\mathrm{H}_{6}^{*}\right) I_{\delta \rho_{1}}^{\rho_{1}}(k) \geq L$.

Then problem (1.1)-(1.2) has at least one positive solution $u$ with $\rho_{1}<\|u\|<\rho_{2}$.

Corollary 3.3 Suppose $\left(\mathrm{H}_{1}\right)-\left(\mathrm{H}_{4}\right)$ hold and $\alpha(t) \geq t$ on $J$. Assume that one of the following conditions holds:

$\left(\mathrm{H}_{7}\right) f^{0}<\phi_{p}(l), I^{0}(k)<q l, I_{\infty}(k) \geq \frac{L}{\delta}$.

$\left(\mathrm{H}_{8}\right) I_{0}(k) \geq \frac{L}{\delta}, f^{\infty}<\phi_{p}(l), I^{\infty}(k)<l$.

Then problem (1.1)-(1.2) has at least one positive solution.

Proof We show that $\left(\mathrm{H}_{7}\right)$ implies $\left(\mathrm{H}_{5}\right)$ and $\left(\mathrm{H}_{6}\right)$. Suppose that $\left(\mathrm{H}_{7}\right)$ holds. Then there exists $0<\rho_{1}<\delta \rho_{2}$ such that

$$
\frac{f(t, u)}{\phi_{p}(u)}<\phi_{p}(l), \quad \frac{I_{k}(u)}{u}<l \quad \text { for } t \in J, 0<u \leq \rho_{1}
$$

and

$$
\frac{I_{k}(u)}{u} \geq \frac{L}{\delta} \quad \text { for } u \geq \delta \rho_{2} .
$$


Hence, we obtain

$$
f(t, u)<\phi_{p}(l) \phi_{p}(u) \leq \phi_{p}(l) \phi_{p}\left(\rho_{1}\right)=\phi_{p}\left(l \rho_{1}\right), \quad I_{k}(u)<l u \leq l \rho_{1} \quad \text { for } t \in J, 0<u \leq \rho_{1}
$$

and

$$
I_{k}(u) \geq \frac{L}{\delta} u \geq \frac{L}{\delta} \delta \rho_{2}=L \rho_{2} \quad \text { for } u \geq \delta \rho_{2} .
$$

Noticing $\delta \rho_{2}<\rho_{2}$, it follows that

$$
I_{k}(u) \geq L \rho_{2} \quad \text { for } \delta \rho_{2} \leq u \leq \rho_{2} .
$$

Therefore, $\left(\mathrm{H}_{5}\right)$ and $\left(\mathrm{H}_{6}\right)$ hold. Hence, by Theorem 3.1, problem (1.1)-(1.2) has at least one positive solution.

Similarly, $\left(\mathrm{H}_{8}\right)$ implies that $\left(\mathrm{H}_{5}^{*}\right)$ and $\left(\mathrm{H}_{6}^{*}\right)$ hold.

Remark 3.2 It is easy to see that Corollary 3.3 holds if we change $\left(\mathrm{H}_{7}\right)$ into the following condition:

$\left(\mathrm{H}_{7}^{*}\right) f^{0}=0, I^{0}(k)=0$, and $I_{\infty}(k)=\infty$

However, we give no information whatsoever on the existence of positive solution for problem (1.1)-(1.2) if we change $\left(\mathrm{H}_{8}\right)$ into the following condition:

$\left(\mathrm{H}_{8}^{*}\right) f^{\infty}=0, I^{\infty}(k)=0$, and $I_{0}(k)=\infty$.

Theorem 3.2 Suppose $\left(\mathrm{H}_{1}\right)-\left(\mathrm{H}_{4}\right), \alpha(t) \geq t$ on J and one of the following conditions holds:

$\left(\mathrm{H}_{9}\right)$ There exist $\rho_{1}, \rho_{2}, \rho_{3} \in(0, \infty)$, with $\rho_{1}<\delta \rho_{2}$ and $\rho_{2}<\rho_{3}$ such that

$$
\begin{array}{ll}
f_{0}^{\rho_{1}}<\phi_{p}(l), & I_{0}^{\rho_{1}}(k)<l \rho_{1}, \quad I_{\delta \rho_{2}}^{\rho_{2}}(k)>\phi_{p}(L), \\
f_{0}^{\rho_{3}}<\phi_{p}(l), & I_{0}^{\rho_{3}}(k)<l \rho_{3} .
\end{array}
$$

$\left(\mathrm{H}_{10}\right)$ There exist $\rho_{1}, \rho_{2}, \rho_{3} \in(0, \infty)$, with $\rho_{1}<\rho_{2}<\rho_{3}$ such that

$$
I_{\gamma \rho_{1}}^{\rho_{1}}(k)>\phi_{p}(L), \quad f_{0}^{\rho_{2}}<\phi_{p}(l), \quad I_{0}^{\rho_{2}}(k)<l \rho_{2}, \quad I_{\gamma \rho_{3}}^{\rho_{3}}(k)>\phi_{p}(L) .
$$

Then problem (1.1)-(1.2) has at least two positive solutions $u_{1}$, $u_{2}$ with $u_{1} \in K \cap\left(\Omega_{\rho_{2}} \backslash \bar{\Omega}_{\rho_{1}}\right)$, $u_{2} \in K \cap\left(\Omega_{\rho_{3}} \backslash \bar{\Omega}_{\rho_{2}}\right)$.

Proof We only consider the condition $\left(\mathrm{H}_{9}\right)$. If $\left(\mathrm{H}_{10}\right)$ holds, then the proof is similar to that of the case when $\left(\mathrm{H}_{9}\right)$ holds.

In fact, it follows from the proof of Theorem 3.1 that

$$
T u \nsupseteq u, \quad u \in K, \quad\|u\|=\rho_{3} .
$$

Applying Lemma 2.4 to (3.1)-(3.3) shows that $T$ has two fixed points $u_{1}$ and $u_{2}$ such that $u_{1} \in K \cap\left(\Omega_{\rho_{2}} \backslash \bar{\Omega}_{\rho_{1}}\right), u_{2} \in K \cap\left(\Omega_{\rho_{3}} \backslash \bar{\Omega}_{\rho_{2}}\right)$. These are the desired distinct positive solutions of problem (1.1)-(1.2). Then proof of Theorem 3.2 is complete. 
Corollary 3.4 Suppose $\left(\mathrm{H}_{1}\right)-\left(\mathrm{H}_{4}\right), \alpha(t) \geq t$ on $J$, and there exists $\rho \in(0, \infty)$ such that one of the following conditions holds:

$\left(\mathrm{H}_{11}\right) \quad 0 \leq f^{0}<\phi_{p}(l), 0 \leq I^{0}(k)<l, I_{\delta \rho}^{\rho}>L$, and $0 \leq f^{\infty}<\phi_{p}(l), 0 \leq I^{\infty}(k)<l$.

$\left(\mathrm{H}_{12}\right) \phi_{p}(L)<f_{0} \leq \infty, L<I_{0}(k) \leq \infty, f_{0}<\phi_{p}(l), I_{0}(k)<l$, and

$$
\phi_{p}(L)<f_{\infty} \leq \infty, \quad L<I_{\infty}(k) \leq \infty
$$

Then problem (1.1)-(1.2) has at least two positive solutions in $K$.

Proof We show that $\left(\mathrm{H}_{11}\right)$ implies $\left(\mathrm{H}_{9}\right)$. It is easy to verify that $0 \leq f^{0}<\phi_{p}(l), 0 \leq I^{0}(k)<l$ imply that there exists $\rho_{1} \in(0, \delta \rho)$ such that

$$
f_{0}^{\rho_{1}}<l, \quad I_{0}^{\rho_{1}}(k)<l .
$$

Let $\eta \in\left(f^{\infty}, \phi_{p}(l)\right)$. Then there exists $r>\eta$ such that $\max _{t \in J} f(t, u) \leq \eta \phi_{p}(u)$ for $u \in[r, \infty)$ since $0 \leq f^{\infty}<\phi_{p}(u)$. Let

$$
\beta=\max \left\{\max _{t \in J} f(t, u): 0 \leq u \leq r\right\} \quad \text { and } \quad \rho_{3}>\max \left\{\phi_{q}\left(\frac{\beta}{\phi_{p}(l)-\eta}\right), \rho\right\} .
$$

Then we have

$$
\max _{0 \leq t \leq 1} f(t, u) \leq \eta \phi_{p}(u)+\beta \leq \eta \phi_{p}\left(\rho_{3}\right)+\beta<\phi_{p}(l) \phi_{p}\left(\rho_{3}\right), \quad \forall u \in\left[0, \rho_{3}\right] .
$$

This implies that $f_{0}^{\rho_{3}} \leq \phi_{p}(l)$. Similarly, from $0 \leq I_{k}^{\infty}<l$, we have $I_{0}^{\rho_{3}}(k) \leq l \rho_{3}$. Hence, $\left(\mathrm{H}_{11}\right)$ implies $\left(\mathrm{H}_{9}\right)$. Similarly $\left(\mathrm{H}_{12}\right)$ implies $\left(\mathrm{H}_{10}\right)$, and the corollary is proved.

Theorem 3.2 can be generalized and we obtain many solutions.

Theorem 3.3 Suppose that $\left(\mathrm{H}_{1}\right)-\left(\mathrm{H}_{3}\right)$ hold and $\alpha(t) \geq t$ on J. Then we have the following assertions.

(1) If there exists $\left\{\rho_{i}\right\}_{i=1}^{2 m_{0}} \subset(0, \infty)$ with $\rho_{1}<\delta \rho_{2}<\rho_{2}<\rho_{3}<\delta \rho_{4}<\cdots<\rho_{2 m_{0}}$ such that

$$
f_{0}^{\rho_{2 m-1}}<\phi_{p}(l), \quad I_{0}^{\rho_{2 m-1}}(k)<l \rho_{2 m-1}, \quad I_{\delta \rho_{2 m}}^{\rho_{2 m}}(k)>L, \quad m=1,2, \ldots, m_{0},
$$

then problem (1.1)-(1.2) has at least $2 m_{0}$ solutions in $K$.

(2) If there exists $\left\{\rho_{i}\right\}_{i=1}^{2 m_{0}} \subset(0, \infty)$ with $\rho_{1}<\rho_{2}$ and

$\rho_{2}<\delta \rho_{3}<\rho_{3}<\rho_{4}<\delta \rho_{5}<\cdots<\rho_{2 m_{0}+2}$ such that

$$
I_{\delta \rho_{2 m-1}}^{\rho_{2 m-1}}(k)>L, \quad f_{0}^{\rho_{2 m}}<\phi_{p}(l), \quad I_{0}^{\rho_{2 m}}<l \rho_{2 m}, \quad m=1,2, \ldots, m_{0}
$$

then problem (1.1)-(1.2) has at least $2 m_{0}-1$ solutions in $K$.

\section{Further results on twin solutions for the case of $\alpha(t) \geq t$ on $J$}

In the previous section, we have obtained some results on the existence of at least two positive solutions for problem (1.1)-(1.2). In this section, we will further discuss the existence of two positive solutions for problem (1.1)-(1.2) under the more general case. 
For $u \in K$, we define the nonnegative increasing continuous functionals $\theta, \theta_{1}$, and $\theta_{2}$ by

$$
\begin{aligned}
& \theta=\min _{t \in[\xi, 1]} u(t)=u(1), \\
& \theta_{1}=\max _{t \in[0, \xi]} u(t)=u(0)
\end{aligned}
$$

and

$$
\theta_{2}=\max _{t \in[0,1]} u(t)=u(0)
$$

It is easy to see that, for each $u \in K$,

$$
\theta(u) \leq \theta_{1}(u)=\theta_{2}(u)
$$

In addition, for each $u \in K, \theta(u)=u(1) \geq \delta\|u\|$, which implies that

$$
\|u\| \leq \frac{1}{\delta} \theta(u) \quad \text { for all } u \in K
$$

Finally, we also note that

$$
\theta_{1}(\lambda u)=\lambda \theta_{1}(u), \quad 0 \leq \lambda \leq 1 \text { and } u \in \partial K\left(\theta_{1}, r\right) \text { for } r>0 .
$$

For notational convenience, we denote

$$
N=\frac{\sigma_{1}}{1-\sigma}, \quad N_{1}=\frac{n+\gamma}{1-\sigma}
$$

We now present the results in this section.

Theorem 4.1 Suppose that there exist constants $0<a<b<c$ such that

$$
0<a<\frac{N}{N_{1}} b<\frac{\delta N}{N_{1}} c
$$

Assume $f$ and $I_{k}$ satisfy the following conditions:

(C $\left.I_{1}\right)(u)>\frac{c}{N}$ for $u \in\left[c, \frac{c}{\delta}\right]$;

$\left(C_{2}\right) f(t, u) \leq \phi_{p}\left(\frac{b}{N_{1}}\right)$ for $(t, u) \in J \times\left[0, \frac{b}{\delta}\right], I_{k}(u) \leq \frac{b}{N_{1}}$ for $u \in\left[0, \frac{b}{\delta}\right]$;

$\left(\mathrm{C}_{3}\right) I_{k}(u)>\frac{a}{N}$ for $u \in[\delta a, a]$.

Then problem (1.1)-(1.2) has at least two positive solutions $u_{1}$ and $u_{2}$ such that

$$
a<\theta_{2}\left(u_{1}\right) \text { with } \theta_{1}\left(u_{1}\right)<b \text { and } b<\theta_{1}\left(u_{2}\right) \quad \text { with } \theta\left(u_{2}\right)<c \text {. }
$$

Proof By the definition of the operator $T$ and its properties, it suffices to show that the conditions of Lemma 2.5 hold with respect to $T$.

We first show that if $u \in \partial K(\theta, c)$, then $\theta(T u)>c$. 
In fact, if $u \in \partial K(\theta, c)$, then $\theta(u)=\min _{t \in[\xi, 1]} u(t)=u(1)=c$. Since $u \in K$, one can get $u(t) \geq c$ for $t \in[\xi, 1]$. Noticing (4.2), we have

$$
c \leq u(t) \leq \frac{1}{\delta} c, \quad t \in[\xi, 1]
$$

As a consequence of $\left(\mathrm{C}_{1}\right)$

$$
I_{k}(u(t))>\frac{c}{N} \quad \text { for } t \in[\xi, 1]
$$

Also, $T u \in K$, and hence we get

$$
\begin{aligned}
\theta(T u)= & \min _{t \in[\xi, 1]}(T u)(t)=(T u)(1) \\
= & \frac{1}{1-\sigma}\left\{\int_{0}^{1} g(t)\left[\int_{1}^{1} \phi_{q}\left(\int_{0}^{s} \omega(r) f(r, u(\alpha(r))) d r\right) d s\right] d t\right. \\
& \left.+\int_{0}^{1} g(t)\left(\sum_{t \leq t_{k}} I_{k}\left(u\left(t_{k}\right)\right)\right) d t\right\} \\
& +\int_{1}^{1} \phi_{q}\left(\int_{0}^{s} \omega(r) f(r, u(\alpha(r))) d r\right) d s+\sum_{t \leq t_{k}} I_{k}\left(u\left(t_{k}\right)\right) \\
= & \frac{1}{1-\sigma} \int_{0}^{1} g(t)\left(\sum_{t \leq t_{k}} I_{k}\left(u\left(t_{k}\right)\right)\right) d t+\sum_{t \leq t_{k}} I_{k}\left(u\left(t_{k}\right)\right) \\
\geq & \frac{1}{1-\sigma} \int_{0}^{1} g(t)\left(\sum_{t \leq t_{k}} I_{k}\left(u\left(t_{k}\right)\right)\right) d t \\
\geq & \frac{1}{1-\sigma} \int_{0}^{t_{n}} g(t) I_{n}\left(u\left(t_{n}\right)\right) d t \\
\geq & \frac{1}{1-\sigma} \int_{\xi}^{t_{n}} g(t) I_{n}\left(u\left(t_{n}\right)\right) d t \\
> & \frac{1}{1-\sigma} \int_{\xi}^{t_{n}} g(t) \frac{c}{N} d t \\
= & \frac{1}{1-\sigma} \frac{c}{N} \sigma_{1} \\
= & c .
\end{aligned}
$$

Next, we verify that $\theta_{1}(T u)<b$ for $u \in \partial K\left(\theta_{1}, b\right)$.

So, letting $u \in \partial K\left(\theta_{1}, b\right)$, then

$$
\theta_{1}(u)=\max _{t \in[0, \xi]} u(t)=u(0)=b .
$$

This implies that $0 \leq u(t) \leq b$ for $t \in[0, \xi]$, and since $u \in K$, we also have

$$
u(t) \leq\|u\| \leq \frac{1}{\delta} \theta(u) \leq \frac{1}{\delta} \theta_{1}(u)=\frac{1}{\delta} u(0)=\frac{1}{\delta} b,
$$


which implies that

$$
0 \leq u(t) \leq \frac{1}{\delta} b, \quad t \in J
$$

It follows from $\left(\mathrm{C}_{2}\right)$ that

$$
f(t, u) \leq \phi_{p}\left(\frac{b}{N_{1}}\right) \quad \text { for }(t, u) \in J \times\left[0, \frac{b}{\delta}\right], \quad I_{k}(u) \leq \frac{b}{N_{1}} \quad \text { for } u \in\left[0, \frac{b}{\delta}\right] .
$$

Noticing $T u \in K$, we obtain

$$
\begin{aligned}
(T u)(t)= & \frac{1}{1-\sigma}\left\{\int_{0}^{1} g(t)\left[\int_{t}^{1} \phi_{q}\left(\int_{0}^{s} \omega(r) f(r, u(\alpha(r))) d r\right) d s\right] d t\right. \\
& \left.+\int_{0}^{1} g(t)\left(\sum_{t \leq t_{k}} I_{k}\left(u\left(t_{k}\right)\right)\right) d t\right\} \\
& +\int_{t}^{1} \phi_{q}\left(\int_{0}^{s} \omega(r) f(r, u(\alpha(r))) d r\right) d s+\sum_{t \leq t_{k}} I_{k}\left(u\left(t_{k}\right)\right) \\
\leq & \frac{1}{1-\sigma}\left\{\int_{0}^{1} g(t)\left[\int_{0}^{1} \phi_{q}\left(\int_{0}^{s} \omega(r) f(r, u(\alpha(r))) d r\right) d s\right] d t\right. \\
& \left.+\int_{0}^{1} g(t)\left(\sum_{k=1}^{n} I_{k}\left(u\left(t_{k}\right)\right)\right) d t\right\} \\
& +\int_{0}^{1} \phi_{q}\left(\int_{0}^{s} \omega(r) f(r, u(\alpha(r))) d r\right) d s+\sum_{k=1}^{n} I_{k}\left(u\left(t_{k}\right)\right) \\
= & \frac{1}{1-\sigma} \int_{0}^{1} \phi_{q}\left(\int_{0}^{s} \omega(r) f(r, u(\alpha(r))) d r\right) d s+\frac{1}{1-\sigma} \sum_{k=1}^{n} I_{k}\left(u\left(t_{k}\right)\right) \\
\leq & \frac{1}{1-\sigma} \int_{0}^{1} \phi_{q}\left(\int_{0}^{s} \omega(r) \phi_{p}\left(\frac{b}{N_{1}}\right) d r\right) d s+\frac{1}{1-\sigma} \sum_{k=1}^{n} \frac{b}{N_{1}} \\
= & \frac{1}{1-\sigma} \frac{b}{N_{1}} \int_{0}^{1} \phi_{q}\left(\int_{0}^{s} \omega(r) d r\right) d s+\frac{1}{1-\sigma} \sum_{k=1}^{n} \frac{b}{N_{1}} \\
= & \frac{1}{1-\sigma} \frac{b}{N_{1}} \phi_{q}\left(\int_{0}^{1} \omega(r) d r\right)+\frac{1}{1-\sigma} n \frac{b}{N_{1}} \\
= & \frac{1}{1-\sigma} \frac{b}{N_{1}} \gamma+\frac{1}{1-\sigma} n \frac{b}{N_{1}} \\
= & (n+\gamma)
\end{aligned}
$$

Finally, we prove that $K\left(\theta_{2}, a\right) \neq \emptyset$ and $\theta_{2}(T u)>a$ for all $u \in \partial K\left(\theta_{2}, a\right)$. In fact, the constant $\frac{a}{3} \in K\left(\theta_{2}, a\right)$. Moreover, for $u \in \partial K\left(\theta_{2}, a\right)$, we have

$$
\theta_{2}(u)=\max _{t \in J} u(t)=u(0)=a,
$$


which implies that

$$
\delta a \leq u(t) \leq a, \quad t \in[\xi, 1]
$$

Using assumption $\left(C_{3}\right)$, we have

$$
I_{k}(u(t))>\frac{a}{N}, \quad t \in[\xi, 1] .
$$

As before $T u \in K$, and so

$$
\begin{aligned}
\theta_{2}(T u)= & \max _{t \in J}(T u)(t)=(T u)(0) \\
= & \frac{1}{1-\sigma}\left\{\int_{0}^{1} g(t)\left[\int_{0}^{1} \phi_{q}\left(\int_{0}^{s} \omega(r) f(r, u(\alpha(r))) d r\right) d s\right] d t\right. \\
& \left.+\int_{0}^{1} g(t)\left(\sum_{t \leq t_{k}} I_{k}\left(u\left(t_{k}\right)\right)\right) d t\right\} \\
& +\int_{0}^{1} \phi_{q}\left(\int_{0}^{s} \omega(r) f(r, u(\alpha(r))) d r\right) d s+\sum_{t \leq t_{k}} I_{k}\left(u\left(t_{k}\right)\right) \\
\geq & \frac{1}{1-\sigma} \int_{0}^{1} g(t)\left(\sum_{t \leq t_{k}} I_{k}\left(u\left(t_{k}\right)\right)\right) d t+\sum_{t \leq t_{k}} I_{k}\left(u\left(t_{k}\right)\right) \\
\geq & \frac{1}{1-\sigma} \int_{0}^{1} g(t)\left(\sum_{t \leq t_{k}} I_{k}\left(u\left(t_{k}\right)\right)\right) d t \\
\geq & \frac{1}{1-\sigma} \int_{0}^{t_{n}} g(t) I_{n}\left(u\left(t_{n}\right)\right) d t \\
\geq & \frac{1}{1-\sigma} \int_{\xi}^{t_{n}} g(t) I_{n}\left(u\left(t_{n}\right)\right) d t \\
> & \frac{1}{1-\sigma} \int_{\xi}^{t_{n}} g(t) \frac{a}{N} d t \\
= & \frac{1}{1-\sigma} \frac{a}{N} \sigma_{1} \\
= & a .
\end{aligned}
$$

Thus, by Lemma 2.5, there exist at least two fixed points of $T$ which are positive solutions $u_{1}$ and $u_{2}$, belonging to $\overline{K(\theta, c)}$, of problem (1.1)-(1.2) such that

$$
a<\theta_{2}\left(u_{1}\right) \quad \text { with } \theta_{1}\left(u_{1}\right)<b \quad \text { and } \quad b<\theta_{1}\left(u_{2}\right) \quad \text { with } \theta\left(u_{2}\right)<c \text {. }
$$

We remark that the condition $\left(\mathrm{C}_{2}\right)$ in Theorem 4.1 can be replaced by the following conditions:

$\left(C_{2}\right)^{\prime} f_{0}^{b} \leq \phi_{p}\left(\frac{1}{N_{1}}\right), I_{0}^{b}(k) \leq \frac{1}{N_{1}} ;$

$\left(C_{2}\right)^{\prime \prime} f^{0} \leq \phi_{p}\left(\frac{1}{N_{1}}\right), I^{0}(k) \leq \frac{1}{N_{1}}$.

Corollary 4.1 If the condition $\left(\mathrm{C}_{2}\right)$ in Theorem 4.1 is replaced by $\left(\mathrm{C}_{2}\right)^{\prime}$ and $\left(\mathrm{C}_{2}\right)^{\prime \prime}$, respectively, then the conclusion of Theorem 4.1 also holds. 
Proof It follows from the proof of Theorem 4.1 that Corollary 4.1 holds.

\section{Triple solutions for the case of $\alpha(t) \geq t$ on $J$}

In this section, we shall study the existence of three positive solutions of problem (1.1)(1.2). Let the nonnegative continuous concave functional $\psi(u): K \rightarrow[0, \infty)$ be defined by

$$
\psi(u)=\min _{t \in[\xi, 1]} u(t)=u(1), \quad u \in K
$$

Note that for $u \in K, \psi(u) \leq\|u\|$.

Theorem 5.1 Suppose that there exist constants $0<d<a<\frac{a}{\delta} \leq c$ such that

$\left(\mathrm{A}_{1}\right) f(t, u) \leq \phi_{p}\left(\frac{d}{N_{1}}\right)$ for $(t, u) \in J \times[0, d], I_{k}(u) \leq \frac{d}{N_{1}}$ for $u \in[0, d]$;

$\left(\mathrm{A}_{2}\right) I_{k}(u) \geq \frac{a}{N}$ for $u \in\left[a, \frac{a}{\delta}\right]$

$\left(\mathrm{A}_{3}\right) f(t, u) \leq \phi_{p}\left(\frac{c}{N_{1}}\right)$ for $(t, u) \in J \times[0, c], I_{k}(u) \leq \frac{c}{N_{1}}$ for $u \in[0, c]$.

Then problem (1.1)-(1.2) has at least three positive solutions $u_{1}, u_{2}$, and $u_{3}$ such that

$$
\left\|u_{1}\right\|<d, \quad a<\psi\left(u_{2}\right) \quad \text { and } \quad u_{3}>d \quad \text { with } \psi\left(u_{3}\right)<a .
$$

Proof By the definition of the operator $T$ and its properties, it suffices to show that the conditions of Lemma 2.6 hold with respect to $T$.

For convenience, we denote $b=\frac{a}{\delta}$.

We first show that if there exists a positive number $r$ such that $f(t, u) \leq \phi_{p}\left(\frac{r}{N_{1}}\right)$ for $(t, u) \in$ $J \times[0, r], I_{k}(u) \leq \frac{r}{N_{1}}$ for $u \in[0, r]$, then $T: \bar{K}_{r} \rightarrow K_{r}$.

Indeed, if $u \in \bar{K}_{r}$, then

$$
\begin{aligned}
& (T u)(t)=\frac{1}{1-\sigma}\left\{\int_{0}^{1} g(t)\left[\int_{t}^{1} \phi_{q}\left(\int_{0}^{s} \omega(r) f(r, u(\alpha(r))) d r\right) d s\right] d t\right. \\
& \left.+\int_{0}^{1} g(t)\left(\sum_{t \leq t_{k}} I_{k}\left(u\left(t_{k}\right)\right)\right) d t\right\} \\
& +\int_{t}^{1} \phi_{q}\left(\int_{0}^{s} \omega(r) f(r, u(\alpha(r))) d r\right) d s+\sum_{t \leq t_{k}} I_{k}\left(u\left(t_{k}\right)\right) \\
& \leq \frac{1}{1-\sigma}\left\{\int_{0}^{1} g(t)\left[\int_{0}^{1} \phi_{q}\left(\int_{0}^{s} \omega(r) f(r, u(\alpha(r))) d r\right) d s\right] d t\right. \\
& \left.+\int_{0}^{1} g(t)\left(\sum_{k=1}^{n} I_{k}\left(u\left(t_{k}\right)\right)\right) d t\right\} \\
& +\int_{0}^{1} \phi_{q}\left(\int_{0}^{s} \omega(r) f(r, u(\alpha(r))) d r\right) d s+\sum_{k=1}^{n} I_{k}\left(u\left(t_{k}\right)\right) \\
& =\frac{1}{1-\sigma} \int_{0}^{1} \phi_{q}\left(\int_{0}^{s} \omega(r) f(r, u(\alpha(r))) d r\right) d s+\frac{1}{1-\sigma} \sum_{k=1}^{n} I_{k}\left(u\left(t_{k}\right)\right) \\
& \leq \frac{1}{1-\sigma} \int_{0}^{1} \phi_{q}\left(\int_{0}^{s} \omega(r) \phi_{p}\left(\frac{r}{N_{1}}\right) d r\right) d s+\frac{1}{1-\sigma} \sum_{k=1}^{n} \frac{r}{N_{1}}
\end{aligned}
$$




$$
\begin{aligned}
& =\frac{1}{1-\sigma} \frac{r}{N_{1}} \int_{0}^{1} \phi_{q}\left(\int_{0}^{s} \omega(r) d r\right) d s+\frac{1}{1-\sigma} \sum_{k=1}^{n} \frac{r}{N_{1}} \\
& \leq \frac{1}{1-\sigma} \frac{r}{N_{1}} \phi_{q}\left(\int_{0}^{1} \omega(r) d r\right)+\frac{1}{1-\sigma} n \frac{r}{N_{1}} \\
& =\frac{1}{1-\sigma} \frac{r}{N_{1}} \gamma+\frac{1}{1-\sigma} n \frac{r}{N_{1}} \\
& =\frac{r}{N_{1}(1-\sigma)}(n+\gamma) \\
& =r
\end{aligned}
$$

which implies that $T u \in K_{r}$.

Hence, we have shown that if $\left(\mathrm{A}_{1}\right)$ and $\left(\mathrm{A}_{3}\right)$ hold, then $T$ maps $\bar{K}_{d}$ into $K_{d}$ and $\bar{K}_{c}$ into $K_{c}$.

Next, we verify that $\{u \in K(\psi, a, b): \psi(u)>a\} \neq \emptyset$ and $\psi(T u)>a$ for all $u \in K(\psi, a, b)$.

Take $u(t)=\frac{a+b}{2}, t \in J$. Then

$$
\|u\|=\frac{a+b}{2}<b, \quad \psi(u)=\min _{t \in[\xi, 1]} u(t)=\frac{a+b}{2}>a .
$$

This shows that

$$
\{u \in K(\psi, a, b): \psi(u)>a\} \neq \emptyset .
$$

Therefore, it follows from $\left(\mathrm{A}_{2}\right)$ that

$$
\begin{aligned}
& \psi(T u)= \min _{t \in[\xi, 1]}(T u)(t)=(T u)(1) \\
&= \frac{1}{1-\sigma}\left\{\int_{0}^{1} g(t)\left[\int_{1}^{1} \phi_{q}\left(\int_{0}^{s} \omega(r) f(r, u(\alpha(r))) d r\right) d s\right] d t\right. \\
&\left.+\int_{0}^{1} g(t)\left(\sum_{t \leq t_{k}} I_{k}\left(u\left(t_{k}\right)\right)\right) d t\right\} \\
&+\int_{1}^{1} \phi_{q}\left(\int_{0}^{s} \omega(r) f(r, u(\alpha(r))) d r\right) d s+\sum_{t \leq t_{k}} I_{k}\left(u\left(t_{k}\right)\right) \\
&= \frac{1}{1-\sigma} \int_{0}^{1} g(t)\left(\sum_{t \leq t_{k}} I_{k}\left(u\left(t_{k}\right)\right)\right) d t+\sum_{t \leq t_{k}} I_{k}\left(u\left(t_{k}\right)\right) \\
& \geq \frac{1}{1-\sigma} \int_{0}^{1} g(t)\left(\sum_{t \leq t_{k}} I_{k}\left(u\left(t_{k}\right)\right)\right) d t \\
& \geq \frac{1}{1-\sigma} \int_{0}^{t_{n}} g(t) I_{n}\left(u\left(t_{n}\right)\right) d t \\
& \geq \frac{1}{1-\sigma} \int_{\xi}^{t_{n}} g(t) I_{n}\left(u\left(t_{n}\right)\right) d t \\
& \geq \frac{1}{1-\sigma} \int_{\xi}^{t_{n}} g(t) \frac{a}{N} d t \\
& \sigma_{1} \\
&=
\end{aligned}
$$


Finally, we assert that if $u \in K(\psi, a, c)$ and $\|T u\|>b$, then $\psi(T u)>a$.

Suppose $u \in K(\psi, a, c)$ and $\|T u\|>b$, then

$$
\begin{aligned}
\psi(T u) & =\min _{t \in[\xi, 1]}(T u)(t)=(T u)(1) \\
& \geq \delta\|T u\| \\
& >\delta b=a .
\end{aligned}
$$

To sum up, the hypotheses of Lemma 2.6 hold. Therefore, an application of Lemma 2.6 implies problem (1.1)-(1.2) has at least three positive solutions $u_{1}, u_{2}$, and $u_{3}$ such that

$$
\left\|u_{1}\right\|<d, \quad a<\psi\left(u_{2}\right) \quad \text { and } \quad u_{3}>d \quad \text { with } \psi\left(u_{3}\right)<a .
$$

We remark that the condition $\left(\mathrm{A}_{3}\right)$ in Theorem 5.1 can be replaced by the following conditions:

$\left(\mathrm{A}_{3}\right)^{\prime} f_{0}^{c} \leq \phi_{p}\left(\frac{1}{N_{1}}\right), I_{0}^{c}(k) \leq \frac{1}{N_{1}}$

$\left(\mathrm{A}_{3}\right)^{\prime \prime} f^{0} \leq \phi_{p}\left(\frac{1}{N_{1}}\right), I^{0}(k) \leq \frac{1}{N_{1}}$.

Corollary 5.1 If the condition $\left(\mathrm{A}_{3}\right)$ in Theorem 5.1 is replaced by $\left(\mathrm{A}_{3}\right)^{\prime}$ and $\left(\mathrm{A}_{3}\right)^{\prime \prime}$, respectively, then the conclusion of Theorem 5.1 also holds.

Proof It follows from the proof of Theorem 5.1 that Corollary 5.1 holds.

\section{Case of $\alpha(t) \leq t$ on $J$}

Now we deal with problem (1.1)-(1.3) for the case of $\alpha(t) \leq t$ on $J$. Let $E$ be as defined in Section 2 . We define a cone $K^{*}$ in $E$ by

$$
K^{*}=\left\{u \in E: u \geq 0, \min _{t \in\left[0, \xi^{*}\right]} u(t) \geq \delta^{*}\|u\|\right\}
$$

where $\xi^{*} \in\left(t_{n}, 1\right)$ and

$$
\delta^{*}=\frac{\xi^{*} \int_{0}^{\xi^{*}} t h(t) d t}{\xi^{*}-\xi^{*} \varsigma+\int_{0}^{\xi^{*}} t h(t) d t} .
$$

It is easy to see that $K^{*}$ is a closed convex cone of $E$.

Define $T^{*}: K^{*} \rightarrow E$ by

$$
\begin{aligned}
\left(T^{*} u\right)(t)= & \frac{1}{1-\varsigma}\left\{\int_{0}^{1} g(t)\left[\int_{0}^{t} \varphi_{q}\left(\int_{s}^{1} \omega(r) f(r, u(\alpha(r))) d r\right) d s\right] d t\right. \\
& \left.+\int_{0}^{1} g(t) \sum_{t_{k}<t} I_{k}\left(u\left(t_{k}\right)\right) d t\right\} \\
& +\int_{0}^{t} \varphi_{q}\left(\int_{s}^{1} \omega(r) f(r, u(\alpha(r))) d r\right) d s+\sum_{t_{k}<t} I_{k}\left(u\left(t_{k}\right)\right) .
\end{aligned}
$$

It is clear that $u(t)$ is a positive solution of problem (1.1)-(1.3) if and only of $u$ is a solution of operator equation (6.1).

By analogous methods, we have the following results. 
Lemma 6.1 Assume that $\left(\mathrm{H}_{1}\right)-\left(\mathrm{H}_{4}\right)$ hold. Then problem (1.1)-(1.3) is equivalent to the fixed point problem of $T^{*}$ in $K^{*}$.

Lemma 6.2 Assume that $\left(\mathrm{H}_{1}\right)-\left(\mathrm{H}_{4}\right)$ hold. Then $T^{*}: K^{*} \rightarrow K^{*}$ is completely continuous.

Similar to the proofs in Sections 3-5, we have the following results. For convenience we introduce the following notations:

$$
\begin{aligned}
& f_{\delta^{*} \rho}^{\rho}=\min \left\{\min _{t \in\left[0, \xi^{*}\right]} \frac{f(t, u)}{\phi_{p}(\rho)}: u \in\left[\delta^{*} \rho, \rho\right]\right\}, \quad I_{\delta^{*} \rho}^{\rho}(k)=\min \left\{\frac{I_{k}(u)}{\rho}: u \in\left[\delta^{*} \rho, \rho\right]\right\} \\
& \sigma_{1}^{*}=\int_{t_{1}}^{\xi^{*}} g(s) d s, \quad N^{*}=\frac{\sigma_{1}^{*}}{1-\sigma}, \quad \frac{1}{L^{*}}=\frac{\sigma_{1}^{*}}{1-\sigma} .
\end{aligned}
$$

Single or twin solutions of problem (1.1)-(1.3) for the case $\alpha(t) \leq t$ on $J$ is treated in the following theorems.

Theorem 6.1 Suppose $\left(\mathrm{H}_{1}\right)-\left(\mathrm{H}_{4}\right)$ hold, $\alpha(t) \leq t$ on $J$, and there exist $\rho_{1}, \rho_{2} \in(0, \infty)$ with $\rho_{1}<\delta^{*} \rho_{2}$ such that the following conditions hold:

$\left(\mathrm{H}_{5}\right) \quad f_{0}^{\rho_{1}}<\phi_{p}(l), I_{0}^{\rho_{1}}(k)<l$.

$\left(\mathrm{H}_{6}\right)^{* *} I_{\delta^{*} \rho_{2}}^{\rho_{2}}(k) \geq L^{*}$.

Then problem (1.1)-(1.3) has at least one positive solution $u$ with $\rho_{1}<\|u\| \leq \rho_{2}$.

Proof If $\left(\mathrm{H}_{5}\right)$ holds, then similar to the proof of (3.1) we have

$$
T^{*} u \ngtr u, \quad u \in K^{*}, \quad\|u\|=\rho_{1} .
$$

Considering $\left(\mathrm{H}_{6}\right)^{* *}$, we have $I_{k}(x) \geq L^{*} \rho_{2}, k=1,2, \ldots, n$, for $\delta^{*} \rho_{2} \leq x \leq \rho_{2}, t \in\left[0, \xi^{*}\right]$.

Since $0 \leq \alpha(t) \leq \xi^{*}$ on $J$, it follows from $\delta^{*} \rho_{2} \leq x(t) \leq \rho_{2}$ on $J$ that

$$
\delta^{*} \rho_{2} \leq x(\alpha(t)) \leq \rho_{2} .
$$

Next, we prove that

$$
T^{*} u \not \leq u, \quad u \in K^{*}, \quad\|u\|=\rho_{2} .
$$

In fact, if there exists $u_{0} \in K^{*},\left\|u_{0}\right\|=\rho_{2}$ such that $T^{*} u_{0} \leq u_{0}$, then we have

$$
\begin{aligned}
0 \leq t \in J \Rightarrow u_{0}(t) \geq & \left(T^{*} u_{0}\right)(t) \\
= & \frac{1}{1-\varsigma}\left\{\int_{0}^{1} g(t)\left[\int_{0}^{t} \varphi_{q}\left(\int_{s}^{1} \omega(r) f\left(r, u_{0}(\alpha(r))\right) d r\right) d s\right] d t\right. \\
& \left.+\int_{0}^{1} g(t) \sum_{t_{k}<t} I_{k}\left(u_{0}\left(t_{k}\right)\right) d t\right\} \\
& +\int_{0}^{t} \varphi_{q}\left(\int_{s}^{1} \omega(r) f\left(r, u_{0}(\alpha(r))\right) d r\right) d s+\sum_{t_{k}<t} I_{k}\left(u_{0}\left(t_{k}\right)\right)
\end{aligned}
$$




$$
\begin{aligned}
\geq & \frac{1}{1-\varsigma} \int_{0}^{1} g(t)\left(\sum_{t_{k}<t} I_{k}\left(u_{0}\left(t_{k}\right)\right)\right) d t \\
\geq & \frac{1}{1-\varsigma}\left[\int_{t_{1}}^{t_{2}} g(t) I_{1}\left(t_{1}, u\left(t_{1}\right)\right) d t\right. \\
& +\int_{t_{2}}^{t_{3}} g(t)\left(I_{1}\left(t_{1}, u\left(t_{1}\right)\right)+I_{2}\left(t_{2}, u\left(t_{2}\right)\right)\right) d t+\cdots \\
& \left.+\int_{t_{n}}^{1} g(t)\left(I_{1}\left(t_{1}, u\left(t_{1}\right)\right)+I_{2}\left(t_{2}, u\left(t_{2}\right)\right)+\cdots+I_{n}\left(t_{n}, u\left(t_{n}\right)\right)\right) d t\right] \\
= & \frac{1}{1-\varsigma}\left[\int_{t_{1}}^{1} g(t) I_{1}\left(t_{1}, u\left(t_{1}\right)\right) d t+\int_{t_{2}}^{1} g(t) I_{2}\left(t_{2}, u\left(t_{2}\right)\right) d t\right. \\
& \left.+\cdots+\int_{t_{n}}^{1} g(t) I_{n}\left(t_{n}, u\left(t_{n}\right)\right) d t\right] \\
\geq & \frac{1}{1-\varsigma} \int_{t_{1}}^{1} g(t) I_{1}\left(u_{0}\left(t_{1}\right)\right) d t \\
\geq & \frac{1}{1-\varsigma} \int_{t_{1}}^{\xi^{*}} g(t) I_{1}\left(u_{0}\left(t_{1}\right)\right) d t \\
= & \frac{1}{1-\varsigma} L^{*} \rho_{2} \sigma_{1}^{*} \\
= & \rho_{2}=\left\|u_{0}\right\| .
\end{aligned}
$$

This is also a contraction. Hence (6.3) holds.

Applying (i) of Lemma 2.4 to (6.2) and (6.3) shows that $T^{*}$ has a fixed point $u \in K^{*} \cap$ $\left(\Omega_{\rho_{2}} \backslash \bar{\Omega}_{\rho_{1}}\right)$. Thus, it follows that problem (1.1)-(1.3) has one positive solution $u$ satisfying $\rho_{1}<u(t) \leq \rho_{2}, t \in J$. This gives the proof of the theorem.

Remark 6.1 The method to deal with the impulsive term in the proof of Theorem 6.1 is different from that in the proof of Theorem 3.1.

Theorem 6.2 Suppose $\left(\mathrm{H}_{1}\right)-\left(\mathrm{H}_{4}\right), \alpha(t) \leq t$ on $J$ and one of the following conditions holds:

$\left(\mathrm{H}_{9}\right)^{*} \quad$ There exist $\rho_{1}, \rho_{2}, \rho_{3} \in(0, \infty)$, with $\rho_{1}<\gamma \rho_{2}$ and $\rho_{2}<\rho_{3}$ such that

$$
\begin{array}{ll}
f_{0}^{\rho_{1}}<\phi_{p}(l), & I_{0}^{\rho_{1}}(k)<l \rho_{1}, \quad I_{\delta^{*} \rho_{2}}^{\rho_{2}}(k)>\phi_{p}\left(L^{*}\right), \\
f_{0}^{\rho_{3}}<\phi_{p}(l), & I_{0}^{\rho_{3}}(k)<l \rho_{3} .
\end{array}
$$

$\left(\mathrm{H}_{10}\right)^{*}$ There exist $\rho_{1}, \rho_{2}, \rho_{3} \in(0, \infty)$, with $\rho_{1}<\rho_{2}<\rho_{3}$ such that

$$
I_{\delta^{*} \rho_{1}}^{\rho_{1}}(k)>\phi_{p}\left(L^{*}\right), \quad f_{0}^{\rho_{2}}<\phi_{p}(l), \quad I_{0}^{\rho_{2}}(k)<l \rho_{2}, \quad I_{\delta^{*} \rho_{3}}^{\rho_{3}}(k)>\phi_{p}\left(L^{*}\right) .
$$

Then problem (1.1)-(1.3) has at least two positive solutions $u_{1}$, $u_{2}$ with $u_{1} \in \Omega_{\rho_{2}} \backslash \bar{K}_{\rho_{1}}^{*}, u_{2} \in$ $K_{\rho_{3}}^{*} \backslash \bar{\Omega}_{\rho_{2}}$.

The following theorem deals with further results on twin solutions of problem (1.1)-(1.3) for the case of $\alpha(t) \leq t$ on $J$. 
Theorem 6.3 Assume that $\left(\mathrm{H}_{1}\right)-\left(\mathrm{H}_{4}\right)$ and $\alpha(t) \leq t$ on J. Moreover, suppose that there exist constants $0<a<b<c$ such that

$$
0<a<\frac{N^{*}}{N_{1}} b<\frac{\delta^{*} N^{*}}{N_{1}} c .
$$

Let $f$ and $I_{k}$ satisfy the following conditions:

$\left(C_{1}\right)^{*} I_{k}(u)>\frac{c}{N^{*}}$ for $u \in\left[c, \frac{c}{\delta^{*}}\right]$;

$\left(C_{2}\right)^{*} f(t, u) \leq \phi_{p}\left(\frac{b}{N_{1}}\right)$ for $(t, u) \in J \times\left[0, \frac{b}{\delta^{*}}\right], I_{k}(u) \leq \frac{b}{N_{1}}$ for $u \in\left[0, \frac{b}{\delta^{*}}\right]$;

$\left(C_{3}\right)^{*} I_{k}(u)>\frac{a}{N^{*}}$ for $u \in\left[\delta^{*} a, a\right]$.

Then problem (1.1)-(1.3) has at least two positive solutions $u_{1}$ and $u_{2}$ such that

$$
a<\theta_{2}\left(u_{1}\right) \text { with } \theta_{1}\left(u_{1}\right)<b \text { and } b<\theta_{1}\left(u_{2}\right) \text { with } \theta\left(u_{2}\right)<c \text {. }
$$

Finally we consider triple solutions of problem (1.1)-(1.3) for the case of $\alpha(t) \geq t$ on $J$.

Theorem 6.4 Assume that $\left(\mathrm{H}_{1}\right)-\left(\mathrm{H}_{4}\right)$ and $\alpha(t) \leq t$ on J. Moreover, suppose that there exist constants $0<d<a<\frac{a}{\delta^{*}} \leq c$ such that

( $\left.\mathrm{A}_{1}\right) \quad f(t, u) \leq \phi_{p}\left(\frac{d}{N_{1}}\right)$ for $(t, u) \in J \times[0, d], I_{k}(u) \leq \frac{d}{N_{1}}$ for $u \in[0, d]$;

$\left(\mathrm{A}_{2}\right)^{*} I_{k}(u) \geq \frac{a}{N^{*}}$ for $u \in\left[a, \frac{a}{\delta^{*}}\right]$;

( $\left.\mathrm{A}_{3}\right) \quad f(t, u) \leq \phi_{p}\left(\frac{c}{N_{1}}\right)$ for $(t, u) \in J \times[0, c], I_{k}(u) \leq \frac{c}{N_{1}}$ for $u \in[0, c]$.

Then problem (1.1)-(1.3) has at least three positive solutions $u_{1}, u_{2}$, and $u_{3}$ such that

$$
\left\|u_{1}\right\|<d, \quad a<\psi\left(u_{2}\right) \quad \text { and } \quad u_{3}>d \quad \text { with } \psi\left(u_{3}\right)<a .
$$

Remark 6.2 Minor adjustments to the proof of Theorem 6.1 are only needed to prove that Theorems 6.2-6.4 hold, so we leave the details to the reader.

\section{Competing interests}

The authors declare that they have no competing interests.

\section{Authors' contributions}

XZ completed the main study and carried out the results of this article. MF checked the proofs and verified the calculation. All the authors read and approved the final manuscript.

\section{Author details}

${ }^{1}$ Department of Mathematics and Physics, North China Electric Power University, Beijing, 102206, People's Republic of China. ${ }^{2}$ School of Applied Science, Beijing Information Science \& Technology University, Beijing, 100192, People's Republic of China.

\section{Acknowledgements}

This work is sponsored by the project NSFC (11301178), the Fundamental Research Funds for the Central Universities (2014ZZD10, 2014MS58) and the Scientific Research Common Program of Beijing Municipal Commission of Education (KM201511232018). The authors are grateful to anonymous referees for their constructive comments and suggestions, which has greatly improved this paper.

Received: 28 October 2014 Accepted: 14 April 2015 Published online: 24 April 2015

\section{References}

1. Bobisud, LE: Steady state turbulent flow with reaction. Rocky Mt. J. Math. 21, 993-1007 (1991)

2. Glowinski, R, Rappaz, J: Approximation of a nonlinear elliptic problem arising in a non-Newtonian fluid flow model in glaciology. Math. Model. Numer. Anal. 37, 175-186 (2003)

3. Sánchez, J: Multiple positive solutions of singular eigenvalue type problems involving the one-dimensional p-Laplacian. J. Math. Anal. Appl. 292, 401-414 (2004) 
4. Kajikiya, R, Lee, YH, Sim, I: One-dimensional p-Laplacian with a strong singular indefinite weight. I. Eigenvalue. J. Differ. Equ. 244, 1985-2019 (2008)

5. Kajikiya, R, Lee, YH, Sim, I: Bifurcation of sign-changing solutions for one-dimensional p-Laplacian with a strong singular weight: $p$-superlinear at $\infty$. Nonlinear Anal. 74, 5833-5843 (2011)

6. Iturriaga, L, Sánchez, J: Exact number of solutions of stationary reaction-diffusion equations. Appl. Math. Comput. 216, 1250-1258 (2010)

7. Dai, $G, M a, R$, Lu, Y: Bifurcation from infinity and nodal solutions of quasilinear problems without the signum condition. J. Math. Anal. Appl. 397, 119-123 (2013)

8. Dai, G, Ma, R: Unilateral global bifurcation phenomena and nodal solutions for $p$-Laplacian. J. Differ. Equ. 252, 2448-2468 (2012)

9. Zhang, X, Feng, M: Existence of a positive solution for one-dimensional singular $p$-Laplacian problems and its parameter dependence. J. Math. Anal. Appl. 413, 566-582 (2014)

10. Zhang, X, Ge, W: Impulsive boundary value problems involving the one-dimensional $p$-Laplacian. Nonlinear Anal. 70 1692-1701 (2009)

11. Cabada, A, Tomeček, J: Extremal solutions for nonlinear functional $p$-Laplacian impulsive equations. Nonlinear Anal. $67,827-841$ (2007)

12. Henderson, J, Wang, H: Nonlinear eigenvalue problems for quasilinear systems. Comput. Math. Appl. 49, 1941-1949 (2005)

13. Yang, Z, O'Regan, D: Positive solutions for one-dimensional $p$-Laplacian equations. Math. Comput. Model. $\mathbf{5 5}$, 1942-1950 (2012)

14. Lü, H, O'Regan, D, Zhong, C: Multiple positive solutions for the one-dimensional singular p-Laplacian. Appl. Math. Comput. 133, 407-422 (2002)

15. Agarwal, RP, Lü, H, O'Regan, D: Eigenvalues and the one-dimensional p-Laplacian. J. Math. Anal. Appl. 266, 383-400 (2002)

16. Wang, H: On the number of positive solutions of nonlinear systems. J. Math. Anal. Appl. 281, 287-306 (2003)

17. Bonannao, G, Livrea, R: Multiplicity theorems for the Dirichlet problem involving the $p$-Laplacian. Nonlinear Anal. 54 1-7 (2003)

18. Du, Z, Lin, X, Tisdell, CC: A multiplicity result for $p$-Laplacian boundary value problems via critical points theorem. Appl. Math. Comput. 205, 231-237 (2008)

19. Ding, Y, O'Regan, D: Positive solutions for a second-order $p$-Laplacian impulsive boundary value problem. Adv. Differ. Equ. (2012). doi:10.1186/1687-1847-2012-159

20. Jankowski, T: Positive solutions to third-order impulsive Sturm-Liouville boundary value problems with deviating arguments and one-dimensional p-Laplacian. Dyn. Syst. Appl. 20, 575-586 (2011)

21. Avery, RI: A generalization of the Leggett-Williams fixed-point theorem. Math. Sci. Res. Hot-Line 2, 9-14 (1998)

22. Kajikiya, R, Lee, YH, Sim, I: Bifurcation of sign-changing solutions for one-dimensional $p$-Laplacian with a strong singular weight: $p$-sublinear at $\infty$. Nonlinear Anal. 71, 1235-1249 (2009)

23. Jankowski, T: Positive solutions of one-dimensional $p$-Laplacian boundary value problems for fourth-order differential equations with deviating arguments. J. Optim. Theory Appl. 149, 47-60 (2011)

24. Guo, D, Lakshmikantham, V: Nonlinear Problems in Abstract Cones. Academic Press, New York (1988)

25. Avery, RI, Henderson, J: Two positive fixed points of nonlinear operator on ordered Banach spaces. Commun. Appl. Nonlinear Anal. 8, 27-36 (2001)

26. Leggett, R, Williams, L: Multiple positive fixed points of nonlinear operators on ordered Banach spaces. Indiana Univ Math. J. 28, 673-688 (1979)

\section{Submit your manuscript to a SpringerOpen ${ }^{\ominus}$ journal and benefit from:}

- Convenient online submission

Rigorous peer review

- Immediate publication on acceptance

- Open access: articles freely available online

- High visibility within the field

- Retaining the copyright to your article 